\title{
Steady states and linear stability analysis of precipitation pattern formation at geothermal hot springs
}

\author{
Pak Yuen Chan and Nigel Goldenfeld \\ Department of Physics, University of Illinois at Urbana-Champaign, \\ Loomis Laboratory of Physics, 1110 West Green Street, Urbana, Illinois, 61801-3080.
}

\begin{abstract}
A dynamical theory of geophysical precipitation pattern formation is presented and applied to irreversible calcium carbonate (travertine) deposition. Specific systems studied here are the terraces and domes observed at geothermal hot springs, such as those at Yellowstone National Park, and speleothems, particularly stalactites and stalagmites. The theory couples the precipitation front dynamics with shallow water flow, including corrections for turbulent drag and curvature effects. In the absence of capillarity and with a laminar flow profile, the theory predicts a one-parameter family of steady state solutions to the moving boundary problem describing the precipitation front. These shapes match well the measured shapes near the vent at the top of observed travertine domes. Closer to the base of the dome, the solutions deviate from observations, and circular symmetry is broken by a fluting pattern, which we show is associated with capillary forces causing thin film break-up. We relate our model to that recently proposed for stalactite growth, and calculate the linear stability spectrum of both travertine domes and stalactites. Lastly, we apply the theory to the problem of precipitation pattern formation arising from turbulent flow down an inclined plane, and identify a linear instability that underlies scale-invariant travertine terrace formation at geothermal hot springs.
\end{abstract}

PACS numbers: 05.45.Ra, 87.23.n, 47.54.-r, 89.75.Kd, 47.20.Hw, 47.15.gm, 47.55.np

\section{INTRODUCTION}

Geophysical pattern formation concerns how geological patterns and landscapes are formed as a result of the underlying physical and chemical dynamics. The aim is to predict the static, dynamical and statistical properties of the variety of geological structures formed. Recently studied examples include travertine motifs, namely dams [1, domes [2] and terraces [2, 3, 4, 5], stalactites 6, 7, as well as that of other patterns such as sand dunes [8, 9, black smoker chimneys at hydrothermal vents[10, columnar joints [1] and braided river networks 12 .

This paper focuses on the formation of travertine structures near geothermal hot springs. In such systems, hot spring water emerges from a vent, and deposits calcium carbonate as a mineral generally termed travertine as it degasses carbon dioxide [1, 2, 3, 4. The formation of stalactites in limestone caves, which are also caused by carbonate precipitation, will also be briefly discussed.

The majority of the work done on the subject has focused on the microscopic aspects of the problem, such as the role of biomineralization due to thermophilic microbes [3, 4], the $\mathrm{CO}_{2}$ degassing mechanisms [13, 14], mineral compositions [15, 16] and crystal structure 17. 18. Here we are interested in the formation of macroscopic structures and motifs, such as domes, stalactites, and terraces [2], which are universal, i.e., independent of microscopic details. In addition, we are interested in the resulting patterns and their correlations, rather than absolute rates of growth; accordingly, microscopic mechanisms that contribute to kinetics, including nucleation processes and potential biomineralization effects, are present in our work through the choice of time scale.

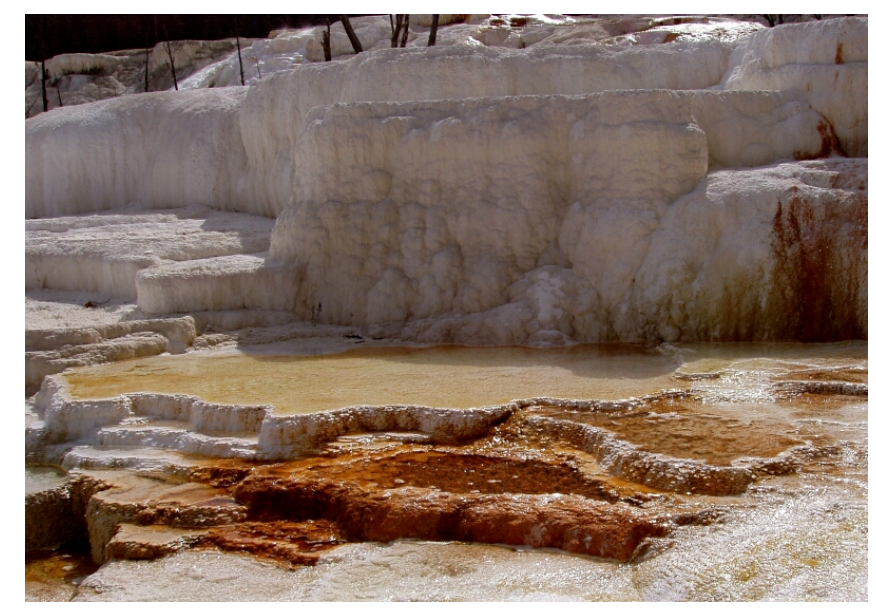

FIG. 1: (Color online) Travertine formation at Angel Terrace, Mammoth Hot Springs, WY, showing a large pond, of order 1 meter in diameter, and smaller features.

There are no extra terms in the equations of motion whose presence can be attributed specially to any one of these microscopic processes.

There are two principal mathematical difficulties encountered in studying these macroscopic structures. First, the problem is highly nonlinear. As the carbonate is precipitated onto the surface, the surface evolves, which then changes the flow path of the fluid, thus affecting how precipitation takes place. This interplay between fluid flow and surface growth leads to a moving-boundary problem, which is mathematically difficult to solve. Second, the problem involves a variety of depositional processes, including solute advection, a complex sequence of chemical reactions, $\mathrm{CO}_{2}$ degassing, as well as mass trans- 
fer between a solid and a liquid. Given that each of these processes is complicated and non-trivial to model on its own, a holistic approach capturing all of them would not be mathematically tractable.

The purpose of this paper is to explore a simplified mathematical formulation of this problem that captures the essential large-scale dynamics. Because of the complexity of the problem, the resulting equations are very complicated, making it difficult, if not impossible, to understand the whole flow system using this approach. It turns out, however, that the equations can be solved analytically under some simple situations, where symmetry can be exploited and simplifications can be made. The formations of domes [2] and stalactites 6, 7] are examples of such situations, as is the pioneering work of Wooding on travertine dams 1. In these systems, there is a thin film of fluid flowing over the motif in a laminar fashion (in the case of domes and stalactites, for example). We will see that these simple motifs are straightforward to calculate in the case that capillary forces can be neglected. If the fluid film becomes too thin, due to its spreading over the surface, contact lines can be formed, resulting in rivulets and the breaking of pure rotational symmetry. In the case of domes, this is manifested in a fluting pattern near the base of the dome2. Such effects are difficult to include analytically, although we have previously shown that they can be captured correctly using a cell dynamical system model[2, and this is discussed in more detail below.

Although we cannot use this analytical theory to study the detailed shapes of the complex landscape of ponds and terraces, we are able to expose the dynamical linear instabilities, whose evolution into the nonlinear regime give rise to the landscape. We will see that the linear stability spectrum, in the absence of capillarity effects, always predicts a positive growth rate. The absence of a length scale arising in this calculation suggests that the actual landscapes might be scale invariant, a conclusion that is reinforced by our studies of the statistical properties of these landscapes using our cell dynamical system model and photographic evidence 2, 19.

The study reported here is a complement to our simulation work 2, 19, 20 implemented as a cell dynamical system. This model has been shown to be capable of describing the actual dynamics 2, not only in the simple cases where the analytical approach is successful, but also in the fully nonlinear regime. For example, it has been shown that this cellular model generically gives rise to a complex, terraced landscape, which is similar to the one observed in the field. The cellular model also makes detailed predictions for the landscape statistics, including the pond area distribution and the distribution of pond anisotropy. In addition, the model successfully predicts that the main mode of pond or terrace growth is uphill pond inundation, a result confirmed by time-lapse photographic studies.

Although seemingly different, both the analytical approach and the cell dynamical system approach incorpo- rate the same physics, and so should be expected to yield identical predictions. In 2 this was tested, by using the cellular model to solve the problem of dome formation. The analytical theory in the absence of surface tension cannot account for the fluting seen away from the vent of domes, because the fluting arises from contact line formation. The analytical theory for domes, as we will discuss in detail below, contains one parameter that sets the scale for the patterns: this scale factor $r_{0}$ is a combination of the upward growth velocity, the mass transfer coefficient describing how material is incorporated into the growing substrate, the flux of water emerging from the vent, the gravitational acceleration and the fluid viscosity. When surface tension effects are included, the capillary length $d_{0}$ must also be included. Thus, our theory is a two parameter theory for the entire range of travertine depositional phenomena. The analytical theory can be used to predict the position on the dome at which capillary effects become important: this must occur at a location that is independent of the ratio $r_{0} / d_{0}$, and hence this critical angle has a prescribed dependence on the underlying parameters which enter into the formula for $r_{0}$. This prediction, arising from the analytical theory, was verified to occur also in the computer simulations of the cellular model[2]. As a result, we conclude that the two formulations are indeed equivalent, and may be used interchangeably depending on which is more suited to the problem at hand.

This paper is organized as follows. In Section II. we derive the equations governing the dynamics of fluid flow coupled to the moving boundary problem describing travertine precipitation. Section III describes the circularly symmetric solutions of these equations, and presents the linear stability analysis of the steady state uniformly translating solutions. We compare our analysis to a similar one 6, 7] that describes the shapes of stalactites in Section [V] and compute the linear stability spectrum of these structures too. We turn in Section VI to a study of turbulent flow down an inclined plane, and calculate the linear stability spectrum for the coupled flow and moving boundary problem, exposing the linear instability that is at the heart of the terraced landscape architecture. We conclude in Section VII.

\section{MODEL FOR PRECIPITATION PATTERN FORMATION}

We consider a stream of water flowing over a terrain, from which calcium carbonate is then, due to geochemical processes to be discussed below, precipitated onto the landscape. The landscape is thus constantly changing in response to the fluid flow. This change of landscape, in turn, affects the flow path of the fluid, which than influences how subsequent precipitation takes place. We derive the governing equations describing both fluid flow and surface growth. We first focus on the surface growth, and related precipitation dynamics, and then move onto 
the fluid flow. These two aspects will be combined to provide the complete description of the system.

\section{A. Surface Growth}

A surface can generally be characterized by the local curvature, $\kappa$. In one dimension, or in cases where symmetry reduces the system to be effectively one dimensional, $\kappa$ is defined by

$$
\kappa=\frac{\partial \theta}{\partial s},
$$

where $\theta$ is an angle between the local tangent of the curve and a fixed axis, and $s$ is the arc length measured from some fixed point on the curve, as shown in Fig. (2). If the normal velocity $v_{n}$ of the surface is prescribed everywhere, then the evolution of the curvature follows the kinematic equation [21, 22, 23]:

$$
\left.\frac{\partial \kappa}{\partial t}\right|_{\theta}=-\kappa^{2}\left(1+\frac{\partial^{2}}{\partial \theta^{2}}\right) v_{n}
$$

The time derivative in the equation is defined with respect to fixed $\theta$. The first term in Eq. (2) describes the change in $\kappa$ due to the change in the overall scale of the object, whereas the second term describes the change in $\kappa$ at a point due to the difference in growth rates in the neighborhood of that point.

Eq. (2) is purely geometrical; for any given function $v_{n}$, the evolution of $\kappa$ is determined. So, physics enters in constructing a realistic and mathematically tractable model for $v_{n}$, which, in the case considered here, depends on water chemistry, surface kinematics, chemical advection and fluid flow state. In carbonate systems, in additional to the $\mathrm{CaCO}_{3}$ concentration, precipitation is mainly controlled by the $\mathrm{CO}_{2}$ concentration (partially reflected in the measured $\mathrm{pH}$ ), which is also influenced by its temperature-dependent solubility in the fluid. As the $\mathrm{pH}$ increases or the temperature decreases, the solvability of $\mathrm{CaCO}_{3}$ decreases and supersaturated $\mathrm{CaCO}_{3}$ will be precipitated onto the surface. While the decrease in temperature is mainly due to heat loss to the environment, the increase in $\mathrm{pH}$ is due to the loss of $\mathrm{CO}_{2}$ by a variety of outgassing mechanisms 13, 14. Although the detailed water chemistry and depositional processes are quite complicated, for the purposes of the present work, it suffices to use a simplification of the governing chemical reactions: $\mathrm{Ca}^{2+}+2 \mathrm{HCO}_{3}^{-} \rightleftharpoons \mathrm{CaCO}_{3}(\mathrm{~s})+\mathrm{H}_{2} \mathrm{O}$ $+\mathrm{CO}_{2}(\mathrm{~g})$. In summary, the system tends to produce more $\mathrm{CaCO}_{3}$ as $\mathrm{CO}_{2}$ concentration decreases through outgassing.

Mass transfer between a fluid and a solid is a complicated problem 1, 24, 25; these nontrivial chemical processes only make it harder. A complete description of the precipitation dynamics, which will give us the normal growth velocity $v_{n}$, involves writing down, in addition to the fluid dynamics equations, advection-reactiondiffusion equations for each chemical and appropriate boundary conditions. Short et al. [6, 7] followed this approach in the study of stalactite formation. What they found, after solving all these equations and taking limits appropriate for the timescales of interest to them, is that $v_{n}$ is proportional to the local fluid thickness, $h$, with all the chemistry entering only into the proportionality constant.

A simple interpretation of this result can be obtained by studying the scales of processes involved in stalactite formation, using parameter values from Ref. 7. The fluid flow is a laminar flow, with Reynold's number about $0.01-1$. The thickness of the flow, $h$, is typically on the order of $10 \mu \mathrm{m}$. The time scale for the concentration of $\mathrm{CaCO}_{3}$ to equilibrate across the layer is thus $h^{2} / D \sim 0.1 \mathrm{~s}$, where $D$ is the diffusion constant. Next, the traversal time, the time for a parcel of fluid to flow along the stalactites, is about 100s. Because only 1 percent of the total $\mathrm{CaCO}_{3}$ mass is precipitated throughout the flow, we can assume that the $\mathrm{CaCO}_{3}$ concentration, and thus the $\mathrm{pH}$, are uniform both across the fluid layer and along the stalactite. The temperature can also be assumed to be constant since the fluid is so thin. The precipitation rate is then controlled only by the $\mathrm{CaCO}_{3}$ available, which is proportional to the thickness of the fluid.

In other carbonate systems, such as at travertineforming hot springs, this relation between $v_{n}$ and $h$ does not hold simply due to the fact that the fluid thickness is larger, and the velocity is larger; as a result a turbulent boundary layer is formed near the precipitation front. What happens outside the boundary layer is too distant to affect precipitation near the boundary. In a turbulent flow, instead of depending on $h$, the precipitation front velocity $v_{n}$ depends on the fluid velocity [24, 25]. Wooding [1], in the study of steady-state dam formation, took this into consideration and arrived at the conclusion that $v_{n}$ is directly proportional to the depth-averaged tangential fluid velocity, $U$, i.e.

$$
v_{n}=G U
$$

where $G$ is a mass transfer coefficient depending on water chemistry and spectral features of the turbulent flow 24, 25]. For present purposes, the functional form of $G$ is not of interest: we shall treat it as a phenomenological parameter, and as we shall see, its role in the theory developed here is to contribute to the characteristic length scale $r_{0}$ of patterns.

To summarize: all the details of water chemistry, including supersaturation, outgassing, solute diffusion, fluid turbulence, temperature and $\mathrm{pH}$, which on their own are complicated processes and are nontrivial to model, enter into the picture only through a mass transfer coefficient, $G$. In principle, $G$ may exhibit spatial fluctuations; however, we shall assume that these are on a scale small compared to the features we are describing, and thus we will consider $G$ to be a constant locally along the flow path. Over the entire geothermal spring system, it is possible that there will be a small spatial variation 


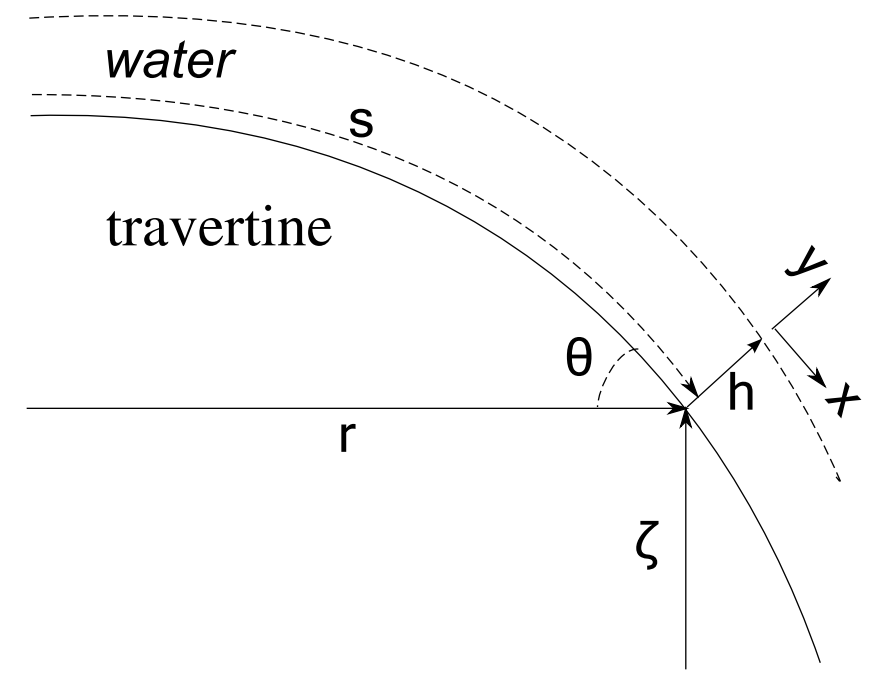

FIG. 2: The coordinate system for the model of fluid flow coupled to precipitation moving boundary dynamics.

in the mean value of $G$, but the weak dependence of $G$ on governing parameters [1, 24, 25] strongly suggests that this can reasonably be neglected.

\section{B. Fluid Dynamics}

A complete description of incompressible fluid dynamics is given by the Navier-Stokes equation

$$
\frac{\partial \vec{u}}{\partial t}+\vec{u} \cdot \nabla \vec{u}=\frac{-1}{\rho} \nabla P+\nu \nabla^{2} \vec{u}+\vec{g}
$$

with $\vec{\nabla} \cdot \vec{u}=0$ for incompressibility, no-slip and stressfree boundary conditions at the solid-liquid and liquidgas interfaces, respectively, where $\vec{u}, \rho, P, \nu$ and $g$ are the fluid velocity, density, pressure, viscosity and gravitational acceleration. We will use the Poiseuille solutions of the Navier-Stokes equations for domes, where the flow is laminar, but for turbulent flows, such as those which form the travertine terraces, we will employ a depthaveraging approximation, in conjunction with the Chézy approximation 26 for hydraulic friction.

Since the spatial scale over which the landscape changes is usually much larger than the fluid thickness, i.e. $h \kappa \ll 1$, we can make use of the shallow water approximation and expand Eq. (4) in powers of $h \kappa$. If we take $\kappa$ to be zero, we arrive at the de Saint-Venant equation 27.

$$
\frac{\partial(U h)}{\partial t}+\frac{\partial\left(U^{2} h\right)}{\partial s}=-g h \frac{\partial h}{\partial s}+g h \sin \theta-\frac{C_{f} U^{2}}{h}
$$

with equation of continuity

$$
\frac{\partial h}{\partial t}+\frac{\partial(U h)}{\partial s}=0
$$

where $C_{f}$ is the Chézy coefficient [26], which empirically describes the energy lost due to turbulence, in a manner consistent with Kolmogorov's 1941 scaling theory of turbulence (K41) 28, 29, and $s$ is the arc length measure from a reference point at the top, as shown in Fig. (2).

The de Saint-Venant equation only holds on flat surfaces. When the surface grows, flow instabilities trigger various patterns to form; and the de Saint-Venant equation is no longer valid. For a general curved surface, the Dressler equation [30, 31] has to be used:

$$
\begin{gathered}
\frac{1}{g} \frac{\partial u_{0}}{\partial t}+\frac{\partial E}{\partial s}=\frac{-C_{f} u^{2}}{g h(1-\kappa h / 2)} \\
(1-\kappa h) \frac{\partial h}{\partial t}+\frac{\partial q}{\partial s}=0
\end{gathered}
$$

where

$$
\begin{gathered}
u(s, n, t)=\frac{u_{0}(s, t)}{1-\kappa n}, \\
E(s, t)=\zeta+h \cos \theta+\frac{p_{h}}{\rho g}+\frac{u_{0}^{2}}{2 g(1-\kappa h)^{2}}, \\
q(s, t)=-\frac{u_{0}}{\kappa} \log (1-\kappa h) .
\end{gathered}
$$

where $\zeta$ is the height of the underlying surface measured from a fixed horizontal axis, as shown in Fig. (2), $p_{h}$ is the pressure head at the fluid surface, $\rho$ is the fluid density, $E$ is the energy density and $q$ is the local flux. When $\kappa$ is set to zero and $\theta$ is small, the Dressler equations reduce to those of de Saint-Venant.

As we have seen, the way fluid flows depends on the landscape it is flowing over, which itself is evolving over time. Now, Eq. (7)-(11) (or Eq. (4)) and Eq. (3) describe these two dynamics, respectively. However, we do not have to consider both dynamics on the same footing because there is a separation of time scales; the rate of fluid flow is on the order of $\mathrm{cm} / \mathrm{sec}$, but the rate of precipitation is on much slower geological scales. The latter is on the order of $1 \mathrm{~mm} /$ day and $1 \mathrm{~cm} /$ century in the cases of Yellowstone travertines 3, 32, 33, and stalactites 7, respectively. Accordingly the fluid flow responds quickly to the landscape, but the landscape responds extremely slowly to the fluid flow. We can then assume that the fluid flow is in its steady state when we discuss the landscape evolution; i.e., we can drop all the time derivatives in the fluid flow equations. This quasi-stationary model will now be used to study the steady states of a variety of geological motifs and their stabilities.

\section{TRAVERTINE DOMES}

\section{A. Steady state}

Our first example is the circularly symmetrical domes found in Yellowstone National Park, as shown in Fig. 
(3a). A number of approximations and simplifications can be made before we proceed. First, the growth rate of these domes is on the order of $1-5 \mathrm{~mm} /$ day and the fluid flow rate is on the order of $1 \mathrm{~mm} / \mathrm{s}$, so we have a separation of time scales. Second, our field observations indicate that the thickness of the fluid film flowing over the domes is very small compared to the curvature of the surface; thus, we make the approximation that the fluid is flowing down a (locally) constant slope. Third, as suggested by the field observations, the domes have a high degree of circular symmetry, so we can assume the solution to be circularly symmetrical and focus only on the radial part of the solution, which is effectively one dimensional. Fourth, the flow is apparently laminar, so we can use the Poiseuille-Hagen profile for the velocity in thin film:

$$
u(y)=\frac{g h^{2} \sin \theta}{2 \nu}\left[2 \frac{y}{h}-\left(\frac{y}{h}\right)^{2}\right],
$$

where $\theta$ is the slope of the surface and $y$ is the transverse coordinate, as shown in Fig. (2). By assuming circular symmetry, $h$ can be related to the axial distance from the vent, $r$, by the conservation of fluid volume:

$$
Q=2 \pi r \int_{0}^{h} u(y) d y=\frac{2 \pi g r h^{3} \sin \theta}{3 \nu},
$$

where $Q$ is the total volumetric flux coming out of the vent. Eq. 12 and 13 can be combined to give

$$
U \equiv \frac{1}{h} \int_{0}^{h} u(y) d y=\left(\frac{\alpha \sin \theta}{r^{2}}\right)^{1 / 3}
$$

where $\alpha \equiv g Q^{2} / 12 \pi \nu$. We will see later that the assumption of laminar flow is self-consistently verified. Putting Eq. (14) into Eq. (2) and using Eq. (3), gives

$$
\left.\frac{\partial \kappa}{\partial t}\right|_{\theta}=-\kappa^{2}\left[1+\frac{\partial^{2}}{\partial \theta^{2}}\right] G\left(\frac{\alpha \sin \theta}{r^{2}}\right)^{1 / 3} .
$$

This is the governing equation for the dome profile. Suggested by the shape of the dome, we seek a solution which steadily translates upwards without a change of shape, i.e., $\left.\partial_{t} \kappa\right|_{\theta}=0$, with velocity $v_{t}$. Eq. (15) gives

$$
G\left(\frac{\alpha \sin \theta}{r^{2}}\right)^{1 / 3}=v_{t} \cos \theta
$$

Rearranging terms gives the shape of the dome as a oneparameter family of curves

$$
\frac{r(\theta)}{r_{0}}=\sqrt{\frac{\sin \theta}{\cos ^{3} \theta}},
$$

where the scale factor $r_{0} \equiv \sqrt{G^{3} \alpha / v_{t}^{3}}$. Eq. 17] is plotted in Fig. (3p). Good agreement is obtained between our theory and the observations below a critical angle $\theta_{c}$. From the fit, and the typical parameter values
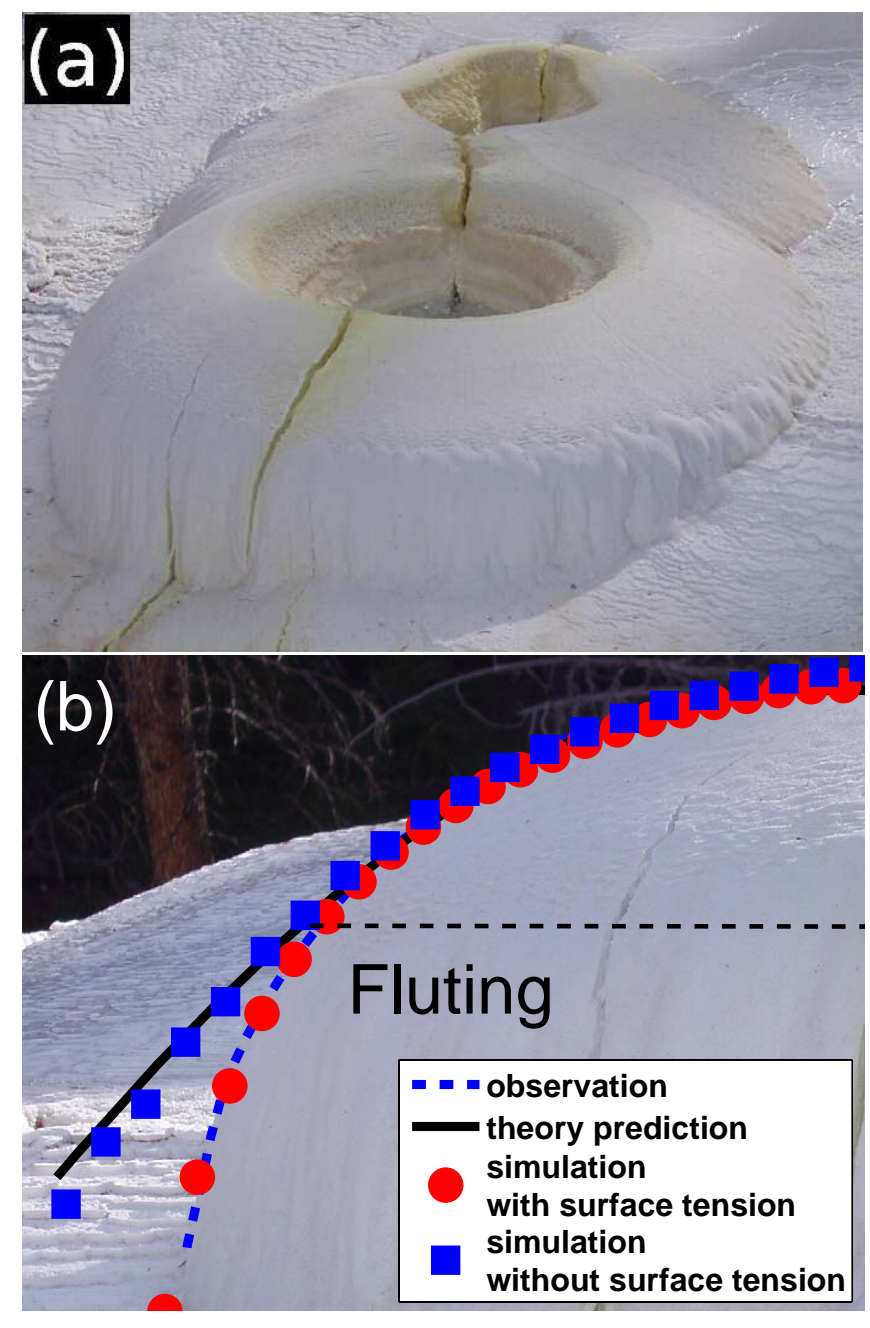

FIG. 3: (Color online) Travertine dome at Mammoth Hot Springs, WY. (a) Dome whose central pond is $50.3 \mathrm{~cm}$ in diameter. (b) Dome profile compared with theory and simulation of Ref. 2. The black curve is the analytical prediction from Eq. (17), using the value $r_{0}=43 \mathrm{~cm}$. The red filled circles show the profile of a simulated dome, including the effects of surface tension. The blue dashed line is a consensus dome profile generated by averaging the dome shown with one other field observation. The blue filled squares show the profile of a simulated dome without surface tension [2].

$G \sim 10^{-8}, v_{t} \sim 1 \mathrm{~mm} /$ day and $Q \sim 1 \mathrm{~cm}^{3} / \mathrm{sec}$, we obtain $U \sim 25 \mathrm{~mm} / \mathrm{sec}$ and $h \sim 1-10 \mathrm{~mm}$, and a Reynolds number, $\operatorname{Re} \equiv U h / \nu \sim 10-100$. The assumption of laminar flow is self-consistently verified.

The agreement between this analysis and observation shows that the growth of the dome is mainly determined by the geometry, because the only $r$ dependence enters through the mass conservation, which is determined by geometry. To see this, suppose that the dome was a one dimensional object. Then, the mass conservation equation, Eq. (13), would become $U h=q_{0}$, for some constant flux $q_{0}$, without any $r$ dependence. Under the same approximation of local flatness, the final equation for $U, \mathrm{Eq}$. 
14, would thus be independent of $r$. We would then not be able to solve for $r$ by substituting $U$ into Eq. (2). In this case, we would have to solve the equations without using the locally-flat approximation. In other words, the fact that we can ignore the details of the flow, by assuming local flatness, to obtain the shape of the domes implies that geometry plays a more important role than fluid flow in the formation of domes.

For angles $\theta>\theta_{c}$, the analytical profile deviates from our field photograph. The point of deviation is associated with an apparent change in the dome morphology, with a fluting pattern superimposed on the dome profile. This is due to the effects of surface tension at the air-water-travertine interface 2. Instead of covering the whole surface uniformly, the fluid separates and covers only a fraction of the surface. Along the wetted surface, the regular growth law still applies and thus the surface grows, until a point at which the difference in heights between the wetted and dry surfaces is so large that the flow changes its path to flow along the dry surface. This process repeats itself and, on average, results in a slower growth when compared with a uniformly-wetted dome, so the theoretical prediction should be higher than the observation for $\theta>\theta_{c}$, as seen in Fig. (3b). The analytical solution for the dome profile neglects surface tension, but leads to a prediction for the scaling dependence of the critical angle on the model parameters [2].

It is not trivial to include surface tension in our analytical model, but its effect can be examined by using the cellular model, in which one can switch on and off surface tension. Fig. (3p), reproduced from Ref. [2] shows the prediction of dome shapes from the cellular model with and without surface tension. It is clear that by appropriate choice of $d_{0}$ the simulation result coincides with the observation when surface tension is present, and agrees with the analytical prediction otherwise. This is direct evidence for the effect of surface tension near fluting.

For completeness, we mention that this is not an artifact of having "enough fitting parameters to fit an elephant". In Ref. [2] was presented a scaling argument for the critical angle at which capillary effects become important. The inclusion of surface tension introduces an additional length scale, namely, the capillary length, $d_{c}$, into the problem. Now, the only other length scale in the problem is $r_{0}=\sqrt{g G^{3} Q^{2} / \nu v_{t}^{3}}$. Since $\theta_{c}$ is dimensionless, it can only depend on the ratio $r_{0} / d_{c}$ and $G$. For a given chemical environment, $G$ is fixed and we are left with the prediction, derived from our analytical solution, that

$$
\theta_{c}=\hat{f}\left(\frac{\sqrt{g Q^{2} / \nu v_{t}^{3}}}{d_{c}}\right),
$$

where $\hat{f}(x)$ is a scaling function. This data collapse, which predicts $\theta$ depends not on the parameters separately, but only on the combination $\sqrt{\left(g Q^{2} / \nu v_{t}^{3}\right)} / d_{c}$, was verified using our discrete cellular model[2], wherein the form of $\hat{f}(x)$ was calculated.

\section{B. Linear stability analysis}

To complete the analysis, we study the stability of the solution, Eq. (17). By following the approach Liu and Goldenfeld used in studying the linear stability of dendritic solidification 34, we consider a perturbed solution, $r(\theta)=\bar{r}(\theta)+\delta r(\theta) e^{\lambda \vec{t}}$, where $\bar{r}(\theta)$ is the solution in Eq. (17) and $\delta r$ is a perturbation. Substituting this into the governing equation, Eq. (15), and expanding in $\delta r$, we obtain

$$
\lambda \frac{d \delta r}{d \theta}+\frac{2 G \alpha^{1 / 3} \cos \theta}{3}\left[1+\frac{d^{2}}{d \theta^{2}}\right] \frac{\delta r \sin ^{1 / 3} \theta}{\bar{r}^{5 / 3}}=0,
$$

where the boundary conditions are

$$
\delta r(0)=0, \quad \delta r\left(\frac{\pi}{2}\right)=0,
$$

for symmetric modes and,

$$
\frac{d(\delta r(0))}{d \theta}=0, \quad \delta r\left(\frac{\pi}{2}\right)=0,
$$

for antisymmetric modes. This is an eigenvalue problem and the spectrum tells us the stability of the solution. It is sufficient to examine the asymptotic behaviors of $\delta r$ for different values of $\lambda$ to extract sufficient information about the stability. Expanding Eq. (19) in small $\theta$ gives

$$
\frac{d^{2} \delta r}{d \theta^{2}}-\frac{1}{\theta} \frac{d \delta r}{d \theta}+\frac{3}{4 \theta^{2}} \delta r=0,
$$

which is independent of $\lambda$ and which possesses power-law solutions of the form $\delta r \sim \theta^{1 / 2}, \theta^{3 / 2}$. These correspond to the symmetric and antisymmetric modes respectively.

The asymptotic behavior in the opposite limit can be studied by making the transformations $g(\theta)=$ $\delta r(\theta) \sqrt{\cot \theta}$ and $x=\tan \theta$, which results in

$$
\frac{d^{2} g(x)}{d x^{2}}+p(x) \frac{d g(x)}{d x}+q(x) g(x)=0,
$$

where

$$
\begin{aligned}
& p(x)=\lambda^{\prime} \sqrt{x\left(1+x^{2}\right)}-\frac{2 x}{1+x^{2}}, \\
& q(x)=\frac{\lambda^{\prime} \sqrt{1+x^{2}}}{2 \sqrt{x}}+\frac{2 x^{2}-1}{\left(1+x^{2}\right)^{2}},
\end{aligned}
$$

and,

$$
\lambda^{\prime} \equiv \frac{3 \alpha^{1 / 6} G^{3 / 2} \lambda}{2 v_{t}^{5 / 2}} .
$$

The asymptotic behaviors of these functions, as $x \rightarrow$ $+\infty$, are

$$
p(x) \sim \lambda^{\prime} x^{3 / 2}+\frac{\lambda^{\prime}}{2 x^{1 / 2}}-\frac{2}{x}+O\left(\frac{1}{x^{5 / 2}}\right),
$$


and,

$$
q(x) \sim \frac{\lambda^{\prime} x^{1 / 2}}{2}+\frac{\lambda^{\prime}}{4 x^{3 / 2}}+\frac{2}{x^{2}}+O\left(\frac{1}{x^{7 / 2}}\right) .
$$

The asymptotic behavior of $g(x)$ as $x \rightarrow+\infty$, for positive values of $\lambda^{\prime}$, can be computed by defining $g(x) \equiv$ $\exp (S(x))$, where $S(x)$ satisfies

$$
\frac{d^{2} S}{d x^{2}}+\left(\frac{d S}{d x}\right)^{2}+p(x) \frac{d S}{d x}+q(x)=0 .
$$

Using the eikonal approximation that $S^{\prime \prime}(x) \ll\left(S^{\prime}(x)\right)^{2}$, which is valid for $x \rightarrow+\infty$, Eq. (29) can be solved asymptotically to give the two linearly independent solutions

$$
S_{1}(x) \sim \frac{-2 \lambda^{\prime}}{5} x^{5 / 2}-\lambda^{\prime} x^{1 / 2}+\ln (x)
$$

and,

$$
S_{2}(x) \sim \frac{-1}{2} \ln (x)
$$

which are equivalent to,

$$
g_{1}(x) \sim \frac{1}{x} \exp \left(\frac{-2 \lambda^{\prime}}{5} x^{5 / 2}-\lambda^{\prime} x^{1 / 2}\right),
$$

and,

$$
g_{2}(x) \sim \frac{1}{\sqrt{x}}+\frac{3}{2 \lambda x^{3}}-\frac{7}{4 \lambda x^{5}}+O\left(\frac{1}{x^{11 / 2}}\right),
$$

where a series expansion in the form of,

$$
g_{2}(x)=\frac{1}{\sqrt{x}} \sum_{n=0}^{\infty} \frac{a_{n}}{x^{n / 2}},
$$

is performed to arrive at Eq. 33.

We see from the asymptotic formula, Eq. [33), that,

$$
\delta r_{2}(x) \equiv \sqrt{x} g_{2}(x) \sim 1+O\left(\frac{1}{x^{5 / 2}}\right)
$$

as $x \rightarrow \infty$ or $\theta \rightarrow \pi / 2$. This means that $\delta r_{2}(\theta)$ does not satisfy the boundary condition, $\delta r(\theta=\pi / 2)=0$. The solution, $\delta r_{1}(\theta)$, is the only solution that satisfies the boundary conditions, Eq. 20p.

To obtain the full eigenfunctions, we use the asymptotic formula, Eq. (32) and (33), as initial conditions and integrate numerically from a large value of $x=c$ ( $c=10$ in this case) back to $x=0$. The Gram-Schmidt orthonormalization procedure is employed to ensure the linear independence of the two eigenfunctions. The eigenfunctions are normalized such that

$$
\int_{0}^{c} \delta r_{i}(x) \delta r_{j}(x) d x=\delta_{i j}
$$
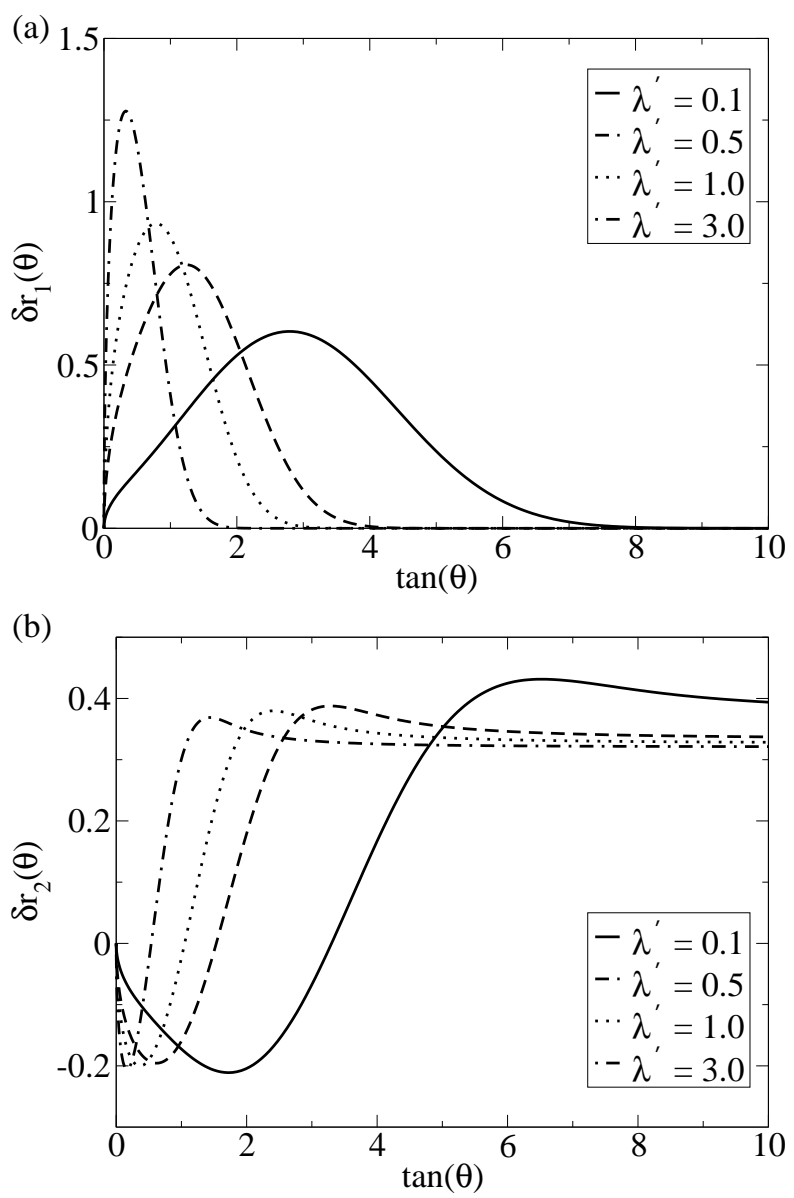

FIG. 4: The eigenfunctions of Eq. 19 for $\lambda^{\prime}=0.1,0.5,1.0$ and 3.0. (a) The first eigenfunction satisfies the boundary conditions for symmetric modes, implying the instability of the dome solution. (b) The second eigenfunction does not satisfy the boundary condition at infinity.

Fig. 4 shows $\delta r_{1}(\theta)$ and $\delta r_{2}(\theta)$ for $\lambda^{\prime}=0.1,0.5,1.0$ and 3.0. From the graph, we confirm that $\delta r_{1}(\theta)$ satisfies the boundary conditions, Eq. 201, while $\delta r_{2}(\theta)$ does not.

Note that $\delta r_{1}(\theta)$ satisfies only the boundary conditions for the symmetric modes, but not the anti-symmetric modes. We need a linear combination of $\delta r_{1}(\theta)$ and $\delta r_{2}(\theta)$ to form a solution that satisfies the latter. But since $\delta r_{2}(\theta)$ does not satisfy the boundary condition at $\theta=\pi / 2$, such a linear combination would not satisfy it either.

To conclude, there are always solutions to Eq. (19) satisfying the boundary conditions for the symmetric modes for every positive value of $\lambda$, i.e., the domes are unconditionally linearly unstable. This seems to be a contradiction with the field observation of domes, which are presumably stable. We will postpone the discussion of this issue to the end of the next section, after we have discussed stalactite formation. 


\section{STALACTITES}

In studying the formations of travertine domes near geothermal hot springs, it helps to study a similar geophysical process, namely, the formation of stalactites, which are cylindrical structures formed by precipitation of calcium carbonate in limestone caves. Here, we will summarize the results Short et al. 6, 7] obtained and apply our formulation to study the stability of stalactites.

\section{A. Steady state}

As discussed earlier, the growth rate of stalactites is directly proportional to the local fluid thickness, $h$. From the field observation, stalactite formation shares the following features with dome formation: They both are circularly symmetrical, formed under a shallow water laminar flow, and can be assumed to be locally flat. So, by using the analysis of dome formation, in particular, from Eq. 13, we have

$$
h=\left(\frac{\beta}{r \sin \theta}\right)^{1 / 3}
$$

where $\beta \equiv 3 \nu Q / 2 \pi g$ is a constant. The dynamical equation, Eq. 2, then becomes

$$
\left.\frac{\partial \kappa}{\partial t}\right|_{\theta}=-\kappa^{2}\left(1+\frac{\partial^{2}}{\partial \theta^{2}}\right)\left[G\left(\frac{\beta}{r \sin \theta}\right)^{1 / 3}\right],
$$

where $G$ depends on water chemistry and the input flux [6, 7]. Following the same strategy employed in the case of travertine domes, we obtain a uniformly translating solution,

$$
r(\theta)=\frac{r_{0}}{\sin \theta \cos ^{3} \theta},
$$

where the tip velocity $v_{t}$ comes in as an integration constant, and the scale $r_{0} \equiv \beta\left(G / v_{t}\right)^{3}$. By defining $\rho \equiv r / r_{0}, z \equiv \zeta / r_{0}$ and using the trigonometric relation $\tan \theta=-d z / d \rho$, we obtain

$$
\frac{z^{\prime}}{\left(1+z^{\prime}\right)^{2}}+\frac{1}{\rho}=0
$$

which is the result derived in Refs. [6, 7].

\section{B. Linear stability analysis}

We study the stability of solution Eq. (39) by introducing a perturbation:

$$
r(\theta)=\bar{r}(\theta)+\delta r(\theta) e^{\lambda t},
$$

where $\bar{r}$ is the unperturbed solution given by Eq. (39) and $\delta r$ is the perturbation. Substituting Eq. 41) into
Eq. (38) and expanding the resulting equation in $\delta r$ gives

$$
\lambda^{\prime} \frac{d \delta r}{d \theta}+\cos \theta\left[1+\frac{d^{2}}{d \theta^{2}}\right]\left(\delta r \sin \theta \cos ^{4} \theta\right)=0,
$$

where $\lambda^{\prime} \equiv 3 G^{3} \lambda / v_{t}^{4}$. We follow the same approach as in the case of the dome and study the asymptotic behaviors of the solutions of Eq. 42. For $\theta \rightarrow 0$, we expand Eq. (42) in $\theta$ and obtain

$$
\lambda^{\prime} \frac{d \delta r}{d \theta}+\left[1+\frac{d^{2}}{d \theta^{2}}\right] \theta \delta r=0
$$

whose solution is given by $r \sim \theta^{\sigma}$, where $\sigma=-1-\lambda$. Because $\sigma<0$ for all $\lambda>0$, the solution diverges as $\theta \rightarrow 0$. This shows that there are no eigenmodes for $\lambda>0$. As a result, we conclude that the steady-state solution Eq. (39) is linearly stable against the class of perturbations considered here.

Let us also look at the asymptotics as $x \rightarrow \infty$ for completeness. Following the strategy employed in the study of dome stability, we make the transformation $g(\theta)=\tan \theta \delta r(\theta)$ and $x=\tan \theta$. Eq. 42 then becomes

$$
\frac{d^{2} g}{d x^{2}}+u(x) \frac{d g}{d x}+v(x) g(x)=0
$$

where

$$
u(x)=\frac{-8 x}{1+x^{2}}+\frac{\lambda^{\prime}\left(1+x^{2}\right)^{3 / 2}}{x},
$$

and,

$$
v(x)=\frac{\lambda^{\prime}\left(1+x^{2}\right)^{3 / 2}}{x^{2}}+\frac{20 x^{2}-5}{\left(1+x^{2}\right)^{2}}+\frac{1}{\left(1+x^{2}\right)^{5 / 2}} .
$$

As $x \rightarrow \infty$,

$$
u(x) \sim \lambda^{\prime} x^{2}+\frac{3 \lambda^{\prime}}{2}-\frac{8}{x}+\frac{3 \lambda^{\prime}}{8 x^{2}}+\frac{8}{x^{3}}+O\left(\frac{1}{x^{4}}\right),
$$

and,

$$
v(x) \sim \lambda^{\prime} x+\frac{3 \lambda^{\prime}}{2 x}+\frac{20}{x^{2}}++\frac{3 \lambda^{\prime}}{8 x^{3}}+O\left(\frac{1}{x^{4}}\right) .
$$

By following the same asymptotic analysis as we did in the last section, we get,

$$
g_{1}(x) \sim \exp \left(\frac{-\lambda^{\prime} x^{3}}{3}-\frac{3 \lambda^{\prime} x}{2}\right)
$$

and

$$
g_{2}(x) \sim \frac{1}{x}+\frac{10}{\lambda^{\prime} x^{4}}-\frac{98}{5 \lambda^{\prime} x^{6}}+O\left(\frac{1}{x^{7}}\right) .
$$

These can be used as the initial conditions to integrate numerically from a large value of $x$, giving the full eigenfunctions. Again, the Gram-Schmidt orthonormalization procedure is employed. The two branches of solutions, $\delta r_{1,2}(\theta)$, are plotted in Fig. 5. They do not satisfy the boundary conditions as they both diverge at $\theta=0$. So the stalactite solution is stable. 

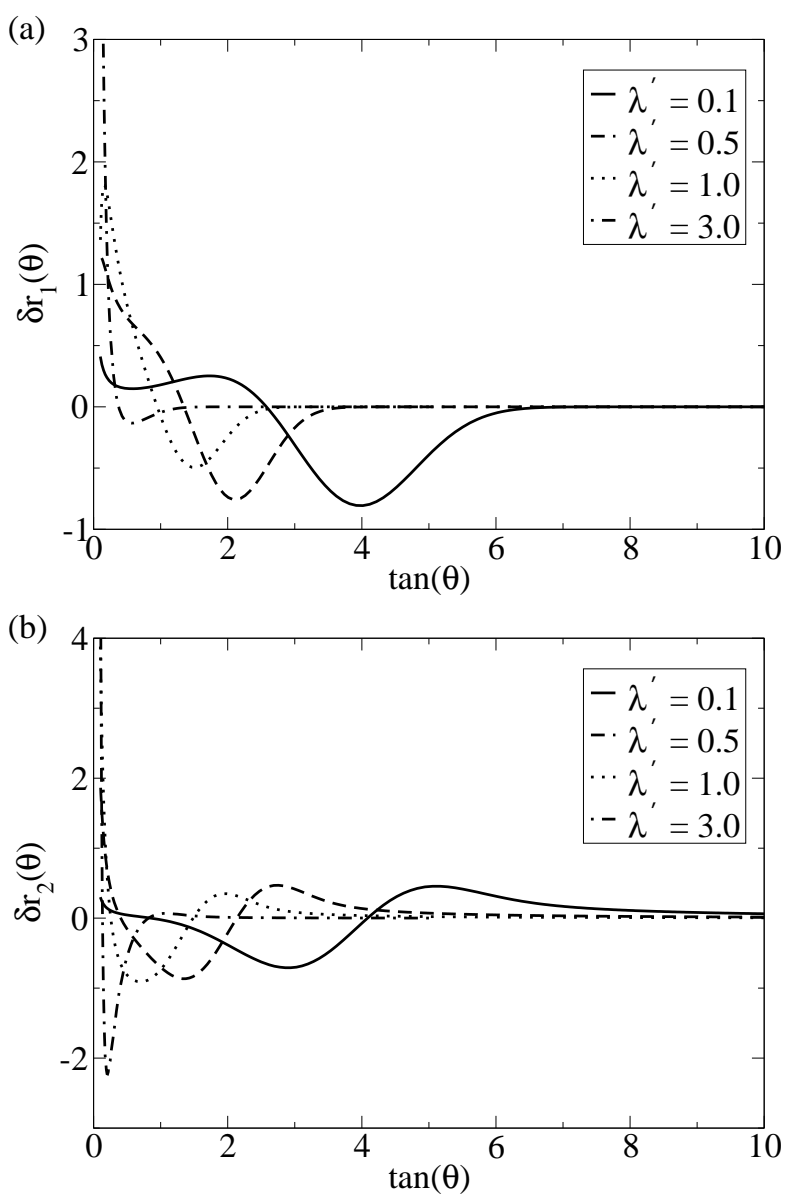

FIG. 5: The eigenfunctions of Eq. 42 for $\lambda^{\prime}=0.1,0.5$, 1.0 and 3.0. These solutions do not satisfy the boundary conditions, as they all diverges at $\theta=0$.

\section{COMPARISON BETWEEN DOMES AND STALACTITES}

We have shown that there is a continuous spectrum of unstable modes for travertine domes, but stalactites, which are formed by an apparently similar process, are predicted to be linearly stable. We need to (a) explain why it is that domes can be observed in the field, and (b) interpret the source of the difference in stability between the two seemingly-related growth motifs. We initially found it surprising that there is a qualitative difference in stability, even though the dynamics of domes and stalactites seem to differ in only relatively minor ways: the growth of domes depends on the depth-averaged fluid velocity whereas the growth of stalactites depends on the fluid thickness. In both cases, the approximation of local flatness is used, so this is unlikely to be the source of the difference.

Our interpretation is that the difference in stability arises from the direction of growth, and as a result, the manner in which surface tension effects correct the zeroth order solutions we have discussed. The direction of growth is important, because it dictates the way in which shape perturbations propagate. For domes growing with sufficiently large $v_{t}$, shape perturbations are advected away from the vent down the body of the dome, in a manner reminiscent of the way in which shape perturbations are advected down the body of a growing dendrite 35. These perturbations may also grow during this process, but the development of this instability is in practice regularized by any non-zero surface tension, leading to contact line formation, film break-up and the formation of rivulets. This heuristic argument is supported by the shape of the linear stability eigenfunctions shown in Fig. 4. For stalactites, on the other hand, the fluid becomes increasingly thick as it flows down towards the tip, and perturbations only increase the growth velocity of the tip, rather than cause growing instabilities away from the tip. Thus, the only place where surface tension is significant is at the tip of the stalactite, where the surface tension holds a water droplet until the droplet becomes too heavy and drops. This dynamics, we believe, mainly contributes to the precipitation rate at the tip, which affects only the growth rate of the whole stalactite. In other words, it only renormalizes the value of $v_{t}$, which, in any case, is a fitting parameter. Surface tension is, therefore, not important in the dynamics of stalactite formation and it should not affect its stability.

Returning now to the case of travertine domes, we conclude that the unstable modes are small near the vent and grow in amplitude near the tail of the dome. This, however, is precisely the region where the film becomes thin and contact line formation can occur, leading to the fluting pattern observed in the real systems. The precipitation rate in this region is also lower, due to the depleted $\mathrm{Ca}^{2+}$ concentration, and this helps stabilizing the domes too. It is possible that the growth of the instabilities predicted here triggers the formation of contact lines and film break-up. Thus, we conclude that the dome is in some sense similar to the problem of dendritic growth, where a smooth tip is followed by a train of sidebranches, widely interpreted to be due to a noise-induced instability [36, 37. It is possible that the full inclusion of surface tension in the model would have as important a role in selection and stability as it does in dendritic growth 38, 39.

\section{DAMMING INSTABILITY}

Having studied the formation of domes and stalactites, we now try to understand some aspects of the large scale morphology of hot spring landscapes. We see in Fig. 1p that the pattern formed is complicated, with ponds of similar shapes but different sizes. Empirical data shows that the distribution of pond sizes indeed follows a power law 20. This scale-invariant pattern hints at an underlying scale-invariant precipitation dynamics, i.e., a dynamics without a characteristic length scale.

It is difficult to predict analytically the statistical prop- 
erties of the landscape, such as the pond size distribution, due to the mathematical complexity of the equations involved. We can, nevertheless, study a simple case of precipitation over a planar slope. By studying the linear stability of this dynamics, we should be able to expose the essential physics of the formation of these scale-invariant patterns. The nonlinear regime of the modeling can be studied using the cellular model we introduced earlier. In this section, we consider a one-dimensional flow down an inclined plane, and evaluate the linear stability spectrum.

The fluid flow in travertine systems is, unlike in the cases of dome and stalactite formations, generally turbulent. It is therefore necessary to use the formulation of Eq. (7)-(11). The turbulent drag leads to a steady flow regime, about which we linearize. Since the angle $\theta$ is the same along a constant slope, it is more convenient to use the arc length, $s$, as the independent variable in the growth equation, so the dynamics of local curvature, $\kappa$, is given by

$$
\left.\frac{\partial \kappa}{\partial t}\right|_{n}=-\left(\kappa^{2}+\frac{\partial^{2}}{\partial s^{2}}\right) G u_{0},
$$

where the subscript $n$ denotes a derivative taken at a point moving along the outward normal of the curve. This, together with the Dressler equation, Eq. (7)-(11), gives the complete description of the system.

We scale the independent variables to their natural units,

$$
t^{\prime}=\frac{U}{R} t, \quad s^{\prime}=\frac{s}{R}, \quad \zeta^{\prime}=\frac{\zeta}{R},
$$

and define the following dimensionless variables,

$$
u_{0}^{\prime}=\frac{u_{0}}{U}, \quad h^{\prime}=\frac{h}{H}, \quad \kappa^{\prime}=R \kappa, \quad \sigma \equiv \frac{H}{R},
$$

where $U, H$ and $R$ are the characteristic scales of the fluid velocity, fluid thickness and the landscape respectively, and $\sigma$ is the ratio between the $H$ and $R$, which is small in the regime of shallow water flow. The governing equations then become (we drop all the primes on the variables for simplicity),

$$
\begin{gathered}
\left.\frac{\partial \kappa}{\partial t}\right|_{n}=-\left(\kappa^{2}+\frac{\partial^{2}}{\partial s^{2}}\right) G u_{0}, \\
\sigma F_{m} \frac{\partial u_{0}}{\partial t}+\frac{\partial E}{\partial s}=\frac{-C_{f} F_{m} u_{0}^{2}}{h\left(1-\frac{\sigma \kappa h}{2}\right)}, \\
(1-\sigma \kappa h) \sigma \frac{\partial h}{\partial t}+\frac{\partial q}{\partial s}=0,
\end{gathered}
$$

with

$$
E=\zeta+\sigma h \cos \theta+\frac{p_{h}}{\rho g}+\frac{\sigma F_{m} u_{0}^{2}}{2(1-\sigma \kappa h)^{2}}
$$

$$
q=\frac{-u_{0}}{\kappa} \ln (1-\sigma \kappa h),
$$

where we defined the Froude number, $F_{m} \equiv U^{2} / g R$.

The uniform solution of this set of equations is given by

$$
\begin{gathered}
\bar{u}_{0}=\sqrt{\frac{\sin \theta}{C_{f} F_{m}}}, \\
\bar{h}=1, \\
\bar{\theta}=\theta_{0} \\
\bar{\kappa}=0,
\end{gathered}
$$

where $\theta_{0}$ is the initial inclination of the slope. The linear stability analysis is performed by adding harmonic perturbations to the solution,

$$
\begin{gathered}
u_{0}=\bar{u}_{0}+\delta u_{0} e^{i p s+\lambda t}, \\
h=1+\delta h e^{i p s+\lambda t}, \\
\theta=\bar{\theta}+\delta \theta e^{i p s+\lambda t}, \\
\kappa \equiv \frac{\partial \theta}{\partial s}=i p \delta \theta e^{i p s+\lambda t},
\end{gathered}
$$

and linearizing the resultant equations to the first order in the perturbations, resulting in three equations for $\delta u_{0}$, $\delta h$ and $\delta \theta$,

$$
\begin{gathered}
i p \lambda \delta \theta=p^{2} G \delta u_{0} \\
\left(\lambda+i p \overline{u_{0}}\right) \delta h+i p \delta u_{0}-\frac{\sigma u_{0} p^{2}}{2} \delta \theta=0 \\
\sigma F_{m} \lambda \delta u_{0}=\delta \theta\left(\cos \bar{\theta}+i p \sigma \sin \bar{\theta}+p^{2} \sigma^{2} \bar{u}_{0}^{2} F_{m}\right) \\
-\delta \theta \frac{C_{f} F_{m} \bar{u}_{0}^{2} \sigma i p}{2} \\
+\delta h\left(-i p \sigma \cos \bar{\theta}+C_{f} F_{m} \bar{u}_{0}^{2}\right) \\
+\delta u_{0}\left(-i p \sigma F_{m} u_{0}-2 C_{f} F_{m} \bar{u}_{0}\right)
\end{gathered}
$$

A single dispersion relation can be obtained by combining all three equations and eliminating $\delta u_{0}, \delta h$ and $\delta \theta$. The result is a cubic equation in $\lambda$,

$$
\lambda^{3}+a_{2}(p) \lambda^{2}+a_{1}(p) \lambda+a_{0}(p)=0,
$$

where

$$
a_{2}(p)=2 i \bar{u}_{0} p+\frac{2 C_{f} \bar{u}_{0}}{\sigma},
$$



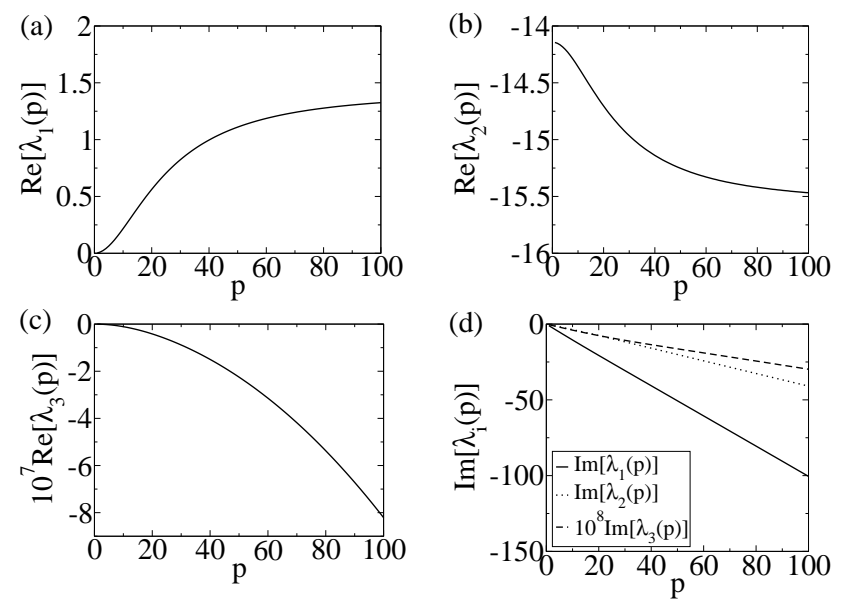

FIG. 6: The damming instability spectrum with parameters $\left(\theta_{0}, G, F_{m}, C_{f}, \sigma\right)=\left(\pi / 6,10^{-8}, 10,0.1,0.01\right)$. (a)-(c) The real parts of the three branches of solutions. The first branch, $\lambda_{1}$, is positive for all $p$, implying that the solution is unconditionally linearly unstable. (d) The imaginary parts of the solutions.

$$
\begin{aligned}
a_{1}(p)= & p^{3} i \sigma \bar{u}_{0}^{2} G \\
& +p^{2}\left(\frac{G \sin \theta}{F_{m}}+\frac{C_{f} \bar{u}_{0}^{2} G}{2}+\frac{\cos \bar{\theta}}{F_{m}}-\bar{u}_{0}^{2}\right) \\
+ & p\left(\frac{i G \cos \bar{\theta}}{\sigma F_{m}}+\frac{3 i C_{f} \bar{u}_{0}^{2}}{\sigma}\right) \\
a_{0}(p)= & p^{4}\left(-\sigma \bar{u}_{0}^{3} G+\frac{\sigma \bar{u}_{0} G \cos \bar{\theta}}{2 F_{m}}\right) \\
& +p^{3}\left(\frac{\left.-i G \bar{u}_{0} \bar{\theta} F_{m}+i G C_{f} \bar{u}_{0}^{3}\right)}{\sin }\right. \\
& +p^{2}\left(\frac{-G \bar{u}_{0} \cos \bar{\theta}}{\sigma F_{m}}\right) .
\end{aligned}
$$

For the parameter set $\left(\theta_{0}, G, F_{m}, C_{f}, \sigma\right)=$ $\left(\pi / 6,10^{-8}, 10,0.1,0.01\right)$, the three roots of the Eq. $70, \lambda_{i}$, are computed numerically and are plotted in Fig. 6. From the graph, we see the first branch of the solutions is always unstable, while the remaining two branches are always stable, implying that the solution is unconditionally linearly unstable. This is the damming instability.

To conclude, we found that the trivial flow down a constant inclined plane is unstable towards all length scales, suggesting that when fully developed into the nonlinear regime, the landscape would have no selected length scale - a surmise in accord with field observations and our cell dynamical system simulations.

\section{CONCLUSION}

By combining fluid dynamics and surface growth kinematics, we formulated a mathematical framework to study geological pattern formation due to carbonate precipitation and applied it to study the formation and stability of a variety of motifs. The theory successfully predicted the shape of observed spherically symmetric domes for angle $\theta$ less than a critical angle $\theta_{c}$. By comparing with results from a cellular model, we showed that the departure of our theoretical prediction from observation for $\theta>\theta_{c}$ is due to the neglect of surface tension. We also showed that domes are linearly unstable towards axisymmetric perturbations, but the instability is manifested in the tail of the dome away from the vent. The instability is masked by the thinning of the fluid film and ultimately the formation of contact lines due to surface tension. This contrasted with the case of stalactites, whose growth forms are linearly stable to axisymmetric perturbations. The difference between the stabilities of the dome and stalactite solutions is attributed to the different geometries and the different role surface tension plays in these two systems.

This formulation cannot predict the complex landscape formed in the fully nonlinear regime, but a linear stability analysis for a one-dimensional flow showed that the apparent scale-invariant landscape is consistent with our equations. In future work, we hope to examine the full two-dimensional instability problem, in order to investigate the dynamics of pond formation, possibly as a transverse morphological instability, akin to meandering in step-flow processes on vicinal surfaces 40 .

\section{Acknowledgments}

We acknowledge stimulating discussions with all the members of the University of Illinois Yellowstone Group, but especially Bruce Fouke, Michael Kandianis and John Veysey, whose expertise, collaboration and review of this manuscript we have greatly enjoyed and appreciated. This material is based upon work supported by the National Science Foundation under Grant No. NSF-EAR0221743. Any opinions, findings, and conclusions or recommendations expressed in this material are those of the authors and do not necessarily reflect the views of the National Science Foundation. 
view Letters 96, 254501 (2006).

[3] B. W. Fouke, J. D. Farmer, D. J. D. Marais, L. Pratt, N. C. Sturchio, and M. K. Discipulo, J. Sed. Res. 70, 265 (2000).

[4] B. W. Fouke, J. Sed. Res. 71, 497 (2001).

[5] O. Hammer, D. Dysthe, and B. Jamtveit, Arxiv preprint physics/0601116 (2006).

[6] M. B. Short, J. C. Baygents, J. W. Beck, D. A. Stone, R. S. T. III, and R. E. Goldstein, Phys. Rev. Lett. 94, 018501 (2005).

[7] M. B. Short, J. C. Baygents, and R. E. Goldstein, Phys. Fluids 17, 083101 (2005).

[8] K. Pye and H. Tsoar, Aeolian sand and sand dunes (Unwin Hyman Boston, 1990).

[9] N. Lancaster, Geomorphology of Desert Dunes (Routledge, 1996).

[10] R. Kerr and J. Turner, Journal of Geophysical Research 101, 25 (1996).

[11] L. Goehring, S. Morris, and Z. Lin, Physical Review E 74, 36115 (2006).

[12] A. Murray and C. Paola, Nature 371, 54 (1994).

[13] J. S. Herman and M. M. Lorah, Chemical Geology 62, 251 (1987).

[14] D. D. Zhang, Y. Zhang, A. Zhu, and X. Cheng, J. Sed. Res. 71, 205 (2001).

[15] I. Barnes and J. R. O'Neil, Geochimica et Cosmochimica Acta 35, 699 (1971).

[16] H. S. Chafetz, P. F. Rush, and N. M. Utech, Sedimentology 38, 107 (1991).

[17] E. Busenberg and L. N. Plummer, in US Geological Survey, Bulletin 1578, edited by F. A. Mumpton (1986), pp. 139-168.

[18] R. W. Renaut and B. Jones, Canadian Journal of Earth Sciences 34, 801 (1996).

[19] J. Veysey and N. Goldenfeld, unpublished.

[20] J. Veysey, Ph.D. thesis, University of Illinois at UrbanaChampaign (2006).

[21] R. Brower, D. Kessler, J. Koplik, and H. Levine, Physical Review Letters 51, 1111 (1983).

[22] E. Ben-Jacob, N. Goldenfeld, J. S. Langer, and G. Schon,
Phys. Rev. Lett. 51, 1930 (1983).

[23] R. C. Brower, D. A. Kessler, J. Koplik, and H. Levine, Phys. Rev. A 29, 1335 (1984).

[24] J. A. Campbell and T. J. Hanratty, AlChE Journal 28, 988 (1982).

[25] J. A. Campbell and T. J. Hanratty, AlChE Journal 29, 215 (1983).

[26] A. Chézy (1776), file No. 847, Ms. 1915 in the library of Ecole des Ponts et Chaussées. English translation in H. Clemens, On the origin of the Chézy formula, Journal Association of Engineering Societies, v. 18, pp. 363-369, (1897).

[27] B. D. Saint-Venant, Comptes Rendus Académie des Sciences, Paris, Tome 73, July (1871).

[28] A. N. Kolmogorov, Dokl. Akad. Nauk. SSSR 30, 299 (1941), [English translation in Proc. R. Soc. London Ser. A 434 (1991)].

[29] K. R. Sreenivasan, Rev. Mod. Phys. 71, S383 (1999).

[30] R. F. Dressler, J. Hydraul. Res. 16, 205 (1978).

[31] N. S. Sivakumaran, R. Hosking, and T. Tingsanchali, J. Fluid Mech. 111, 411 (1981).

[32] I. Friedman, Geochimica et Cosmochimica Acta 34, 1303 (1970).

[33] A. Pentecost, Geol. Mag. 127, 159 (1990).

[34] F. Liu and N. Goldenfeld, Physical Review A 38, 407 (1988).

[35] M. Barber, A. Barbieri, and J. Langer, Physical Review A 36, 3340 (1987).

[36] R. Pieters and J. Langer, Physical Review Letters 56, 1948 (1986).

[37] R. González-Cinca, L. Ramírez-Piscina, J. Casademunt, and A. Hernández-Machado, Phys. Rev. E 63, 051602 (2001).

[38] E. Ben-Jacob, N. Goldenfeld, B. Kotliar, and J. Langer, Physical Review Letters 53, 2110 (1984).

[39] D. Kessler, J. Koplik, and H. Levine, Physical Review A 31, 1712 (1985).

[40] G. Bales and A. Zangwill, Physical Review B 41, 5500 (1990). 